\title{
IoT Device and Service Discovery Framework
}

\author{
Akhileshwari K G, Supriya Salian
}

\begin{abstract}
Internet of Things (IoT) is a unique domain which works on various aspects, which is blanketed, interconnected and thereby enables the humans to interact with the web services and creates a smarter world. There is severe problem in the unified way for discovering IoT devices and services in the present existing infrastructure. The current device centric approach is not in consistent with the growing network. There is no standard method which allows the user to find IoT devices and services in a single framework. Users looking for services will not be aware of potential services available to satisfy their needs. The proposed concept uses unified service discovery broker based architecture using web services. Broker based architecture allows the different providers to register their devices and services at one place and based on the user query, matchching of suitable device and appropriate service will be done and the service data corresponding to the specific query of the user will be provided by using semantic search. Hence, the user can find in a single framework the registered devices and services separately which will be bound together to process the IoT device data stored on cloud. In future, IoT applications would be deployed in several domains like home automation, smart cities, intelligent transport and e-health. Complex service request made by the user can also be resolved using service composition to get the final result.
\end{abstract}

Keywords-IoT; broker based architecture; semantic search; device discovery, service discovery, web service.

\section{INTRODUCTION}

Internet of Things (IoT) is a technology which is formulated by merging several technologies together, such as embedded systems, machine learning, real time analytics and commodity sensors [1]. For enabling the IoT, many traditional fields have their own contributions in it. The traditional fields contain many technologies like wireless sensor network, embedded system, automation, control system and many more. The IoT Domain will work with several things together that would enable human beings to interact with the web services to achieve the goal of creating a smart world. The research and development area of IoT face a host of changes in creating a perfect smart world. IoT works in almost every field in this era such as smart city, smart transportation system, Agriculture, digital health and smart industries and develop the business among many other things. Internet of Things aims at connecting people with the surrounding things, objects and

Revised Manuscript Received on July 22, 2019.

Akhileshwari K G, Department of Computer Science and Engineering, St Joseph Engineering College, Mangaluru, India

E.Mail: akhilakg.bhat@gmail.com

Supriya Salian, Department of Computer Science and Engineering, St

Joseph Engineering College, Mangaluru, India

E.Mail: supriyas@sjec.ac.in places around at any given point of time anywhere in the globe by introducing various new verticals and dimensions.

The IoT has many applications in the areas of commercial, consumer, industrial and infrastructure applications. These applications are divided into several subsections such as elder care, smart home, transportation, medical \& health care, energy management, agriculture, environmental monitoring, building \& home automation and manufacturing and in such other and similar nuances. In all these areas of its application, IoT technologies are successful in significantly reducing the human efforts and have improved the quality of life. There are several areas where intelligent applications are developed, which are not readily available applications. However, the research in the field of IoT shows that, it takes an important part in improving the quality of life by creating a smart, interlinked society.

It is proved that the IoT take part in providing benefits in the field of health and wellness. To monitor person's health condition, so many wearable sensors and devices were already developed and are already in use. The usage of IoT in health application makes the old and elderly, the patients with severe health issues to live independently. The sensors which are installed in the human body continuously measure and monitor the medical parameters. These health care applications can continuously monitor a patient's health condition when he is not under medical supervision or when he is all alone [2]. The IoT sensors used in health monitoring system continuously monitor and record the health conditions of humans. If any abnormalities are detected in patient's health condition, it transmits the warning to a doctor or the caretaker. If the abnormality occurred is minor then, system itself will give some prescription to the patient.

In health a nd wellness domain, many wearable sensors are available to monitor person's health. These typre of sensors are used to meassure different types of parameters such as pulse rate, blood pressure level, heart beat count, temperature of the body, amount of calories burnt, respiration rate, number of steps trodden and blood glucose levels. These wearable devices may be in the form of wristbands, smart watches, smart textiles and monitoring patches.

The problem faced by a service requestor here could be when he enters a query, all the available service URL's will be presented to the service requester and it will be the responsibility of the service requester to search for his needy service.

For example, retrieving the heart beat value can be used to illustrate the solution to the challenge faced in service discovery. The service requester seeking a service will enter the query. Semantic search

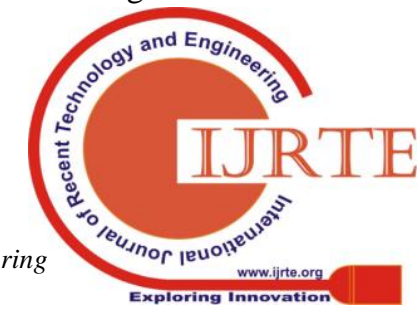


take place to analyze the query to provide appropriate requested service. The semantic search analyze the query in such a way that, it selects the device and service which are stored in the cloud and produce the analyzed heart beat result to the service requester.

\section{A. Semantic Search}

Semantic search is a searching technology which intelligently understands the context of the query, intension of the user and the relationships between the words present in the query [3]. Semantic search engine uses ontology so that meaningful and accurate results will be returned to the user query. Relevant results are provided to the query depending upon the meaning and relations of the words present in the query.

Semantic search is coined from semantic web and is developed on ontology. System of knowledge in the field of computers and information science consists of ontological frameworks for information and factual substance. The semantic search considers several factors to perform the search. For example, when you enter a query such as "Best android phones with 5 inch display" on a semantic search engine, you get a very different result than the traditional search engines. Rather than showing results based on keywords present in the query, semantic search considers context and user's intention of the query and provides the result using some set of attributes.

\section{B. IoT Device Discovery}

The IoT devices are deployed in distributed and heterogeneous environment. Management and the discovery of such IoT devices are quite challenging. Hence, device discovery plays a crucial role in resource usage and management of generated data in a particular available IoT environment. Device discovery constitutes a major challenge for IoT. In order to complete a task, finding useful devices is a must.

\section{IoT Service Discovery}

Service discovery in a given list of named instances containing the desired services allow the client to discover a type of service and a domain name, using standard queries. The main goal of service discovery is to provide browsing and resolving service names. In a last few years, significant attention has been garnered on IoT. The service discovery has brought new challenges and requirements as it used in multiple applications and domains like smart healthcare, IT transportation, smart home etc and is highly dynamic. Hence, it is becoming more and more challenging to discover the desired and suitable services.

\section{Web Service}

Computing technology which is available across many platforms and which offers better communication among many languages and applications in business is known as Web Service. A URI is used to identify a particular Web Service application. XML messaging system is used to discover describe and identify the interfaces and the binding between them. Coordination and computing platform among many businesses and application platform use web service as a part of the service oriented computing. SOAP, XML, WSDL and UDDI etc. are used to access the services, the independent software applications which are available in the web. RESTful Web and SOAP-based web service are widely accepted and are the most popular web services [4].

\section{LITERATURE SURVEY}

Wang and Chow outline the changes required to shift the present IoT framework into IoT service model [5]. They have described a framework which provides the resultant output to the service preferences provided by the user and manage data that is generated and consumed by services. The current framework of existing Auto-ID was modified and developed by using the Client's currently available Physical Mark-up Language architecture and Object Name Service. A phone application which can interact with IoT service discovery framework has also been provided in the said work. Interesting and available services in the locality are notified to the users. However, a lot of drawbacks still exist. Further research and development is required to improve the system performance and user interface. If the devices and services in the smart city are numerous, then latency and bandwidth needs to be practical and scalable for services. Another drawback is in respect of representation of excessive amount of IoT services in an extendable and meaningful way. This shows the difficulty in maintaining data in an effective interest filtering and for privacy.

Aziez et al. used a comparison work in IoT for different approaches used in discovery of services [6]. They studied the shortcomings that are already existing and the available solutions to handle the problem in many ways in IoT service discovery. The description model of IoT service, the service discovery mechanism of IoT, the architecture adopted and the awareness of the context are taken as four perspectives to make and conclude the comparative study. The said comparative study describes problems as well as the solutions that are generated during the discovery service in IoT domain. Classification of IoT discovery service approaches were made based on the mechanism of discovery. Comparison work revealed that to explain the services of IoT formally there were no new standard language. Incorporating the OWLS with WSDL with DPWS or new attributes or COPE services to the traditionally existing web services had to be considered to support the capabilities of devices and to adapt to the features of IoT service.

Datta et al. applied IoT to the concept of smart homes to personalize healthcare [7]. This smart home architecture enables user to get connected, smarter to personalize the healthcare and services in wellness. The physical parameters of wearable sensors are continuously monitored. Along with the wearable sensors, sensors deployed in smart homes also help in better understanding of the user's environment. So this architecture combines sensor measurements from eHealth domain as well as different other domains. Because of the accessibility of different domains in this architecture, for an instance, the room temperature can be adjustable based on human's body temperature level. Semantic web service technology used here to provide actionable intelligence by

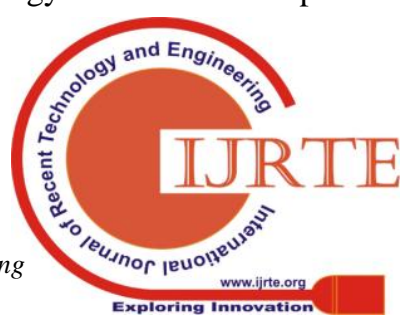


combining sensor data from several other different domains. Different devices which are placed in different domains are discovered, managed and interacted by using Machine-to-Machine (M3) framework. This is the main aim of M3 architecture. Along with this it also provides high level abstract generation framework from sensor data to form healthcare solutions by combining the sensor data from various other different domains.

Fredj et al. proposes an approach which is used to enhance the problem occurred in the discovery of IoT semantic web services, where the architecture uses semantic gateway in dynamic context [8]. In every semantic gateway, for the modification in mechanism leads in service dynamicity uses incremental clustering method. This clustering is enhanced over a period of time by considering number of service and number of clusters in one cluster. The result they got shows that the cost for discovery is invariably same. Also, the comparison work with the approach of non-clustering shows very well optimized result. From the result, it shows that the cost occurred for matching in incremental clustering is negligible compared with the cost occurred in discovery.

Prakash et al. aimed with the IoT architecture which is mainly used for discovery of services [9]. The system architecture used in IoT do the service discovery in every layers of IoT. To create applications of IoT, this architecture includes discovery based on demand and device integration, service integration, application and visualization selected and arranged dynamically.

D'Mello et al. gives a review about different composition techniques and architectures used in composition of dynamic web service [10]. For the web service composition in dynamic way the study includes different techniques. The proposed composition mechanism showed in study uses composition plan that helps to involve web services related to complex service request to complete numerous tasks. The requester's request normally contains a request for either single operation or for multiple operation, these multiple operations are connected through a collection of logical operation by way of web service. So, the composition plan uses various web service together to involve abstract operations from different other web services to generate a proper composition plan. The execution order of one web service depends on the operation of other web service. Some operations involved in web service, assist other operations in the execution to get success instead implementing the business logic itself.

According to the requirements of customer the web service finds devices along with their data and their interaction with other devices to form the goal of IoT. Chirila et al. applied Service Oriented Architecture (SOA) with some standard for web services such as SOAP, WSDL to the devices [11]. The proposed architecture used broker architecture for discovering and recommendation in smart device. The needs of service requester are to match the services which are available by way of discovering web service. The architecture added web service clustering method to reduce the search space from candidate service which relays on similarity metric applied in the web service interface.
Khodadadi et al. proposed architecture for IoT environment to provide a standard and an efficient way to discovery, service composition and the integration of services in scalable manner [12]. The architecture uses Simurgh framework in providing some standard to define, discover and to compose the things with their corresponding services. The framework considers user as a main player and provide efficient results in IoT device discovery with their exposed services. This approach creates ubiquitous environment to establish the communication between the entities of IoT elements. The elements described in the approach uses standard human-and-machine readable files, which provides standard RESTful web APIs to advertised IoT services to find each other easily. Furthermore, the end users can able to achieve their goals by chaining IoT service calls together to make the flow and also the arrangements and combinations of flows are done to get better functionality.

Aziez et al. proposed ontological model to address the problem faced in describing the context to IoT environments and the affects that are generated in the service discovery in IoT [13]. To form the IoT environment the model uses IoT Context to describe the different contexts that are used in main entity. The proposed model contains the advantages such as extensibility, independency in domain and the availability of IoT constraint. Also, the model can be extended, used in some other domains and are able to reuse external ontologies such as GeoNames for location and SSN ontology for sensors.

Moeini et al. developed a routing protocol in semantic basis for the discovery of IoT services [14]. The routing protocol creation aimed in reduction of space requirement in routing. Each routing node holds one routing table to maintain the details of next immediate neighbor and the summary to give next capabilities for further away neighborhood. Coding scheme is used in ontology to reduce the memory requirements that are generated during capability summarization. Coding scheme worked well in reduction in the routing table size so that it is suitable for IoT device with restricted memory. The results from this ontology shows that it produce lower network traffic and also latency for IoT capability lookup when compared with the other existing semantic routing algorithm.

Kim and Lee proposed an echo system by using IoT devices and services for the worldwide use [15]. The proposed open service framework provides opportunities for the device developers to register their devices and API's. By using the registered API's the software developers develop the application or the web programs to connect and control the registered IoT devices and also register them on App store site in the network. The framework also allows service providers to purchase and register IoT devices in open service framework so that, it provide easy management and monitoring of growing number of IoT devices. This frame work provide the advantage for network operator by way of mobile and wireless communication technology and it provides IoT device searching and browsing services to the consumer.

Pandiarajan et al. proposed the semantic web by using Natural Language processing (NLP) to the retrieval of relevant documents [16]. The

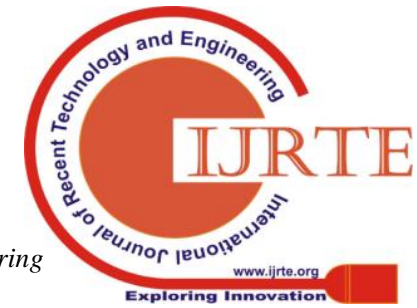


user entered query is analyzed by using NLP in terms of Parts of Speech. The terms which are extracted from the query are compared with domain dictionary to provide the result regarding relevant domain in which the user has interest. Also, the documents which are retrieved based on user entered query are investigated by using NLP to identify the particular domain. Ranking is generated to the documents based on the relevancy to the contents which are present in the query. While comparing this proposed architecture, the time taken for the classification is high.

Ma et al. proposed a plan that combines traditional web service discovery strategy together with the semantic web technology and search engine technology [17]. Lexical analysis along with the grammar analysis is done by using search engine technology. Based on semantic information the matching and scoring are done in semantic web technology. Adding up of semantic information to services and keyword matching at syntax level are take place. For the query, results based on search are listed down and this list provided to the users with the ordering of higher score to lower score. Advantage given by this method is improved service's recall ratio with precision for the strategy of discovering web service.

El-gayar et al. proposed semantic search engine framework by providing new algorithm for ranking the result [18]. The framework involves 4 main phases such as crawling, indexing, ranking and retrieval phase. The two main algorithms considered for the frameworks are enhanced crawling algorithm and efficient proposed ranking algorithm. The content relevant to the forum from the web is crawled by using enhanced crawling algorithm with the minimal overhead. For making the faster retrieval process along with the accurate and easy access to the data proposed ranking algorithm is used also, this algorithm used for here to order and evaluate identical meaningful data.

\section{PROBLEM STATEMENT}

People lack a unified way to discover IoT devices and services in the present existing infrastructure. The current device-centric approach to IoT is incessant with the growing network and software services associated with these devices. There is no standard method where user can find IoT devices and services at one place. This project work aims at creation of device and service registry and also handles the discovery mechanism in order to provide available devices and service by coordinating and creating a match between the available devices and the services as sought by the requester.

Problem Definition: If the user requests for a specific service by way of a query clearly describing his demand, matching devices and services should be made available to the user by using semantic search. Then the corresponding service data should be returned to the user.

Users looking for services will not be aware of potential services available to satisfy their needs. Therefore, proposed architecture uses unified service discovery Broker based architecture using web services. Broker based architecture allows the different providers to register their devices and services at one place. The user need not search for the devices and services separately. This proposed device and service discovery framework, gives the list of available devices and services based on the user query and upon match making between the suitable device and appropriate service, the service data corresponding to the specific query of the user will be provided by using semantic search.

\section{PROPOSED METHODOLOGY}

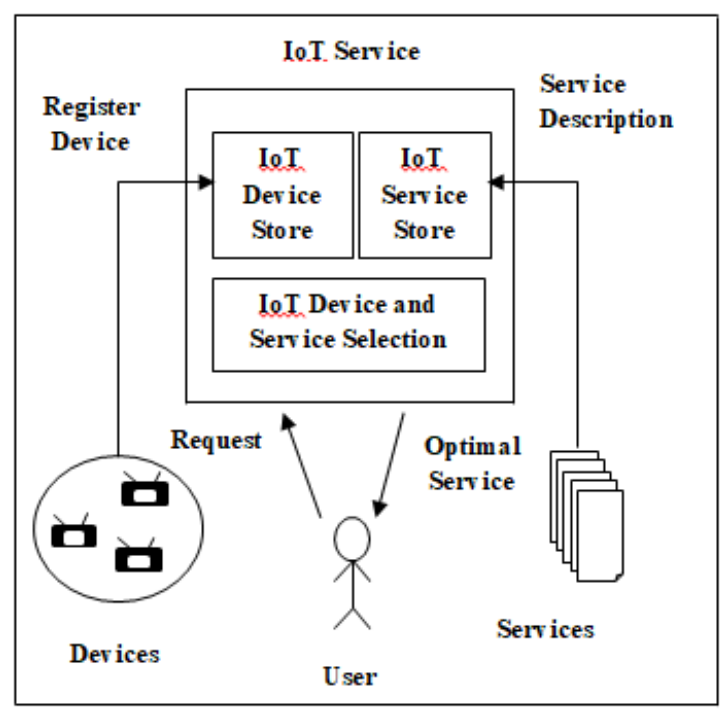

Figure 1: Architectural design

IoT devices of various providers are registered in the IoT device store, which is present in the IoT service broker. The corresponding services required for the devices are registered in the IoT service store by the service providers. On user requesting for a specific device or a specific service, the system architecture will fetch the results from the cloud using keyword search. Subsequently, match making and binding between the devices and services would take place by using semantic search. Finally, the result would be made available to the user. The architectural design of proposed methodology shown in figure1.

\section{A. Internal Architectrue of the IoT Service Broker}

Internal architecture provides the detailed procedure that runs inside an IoT Service Broker. 


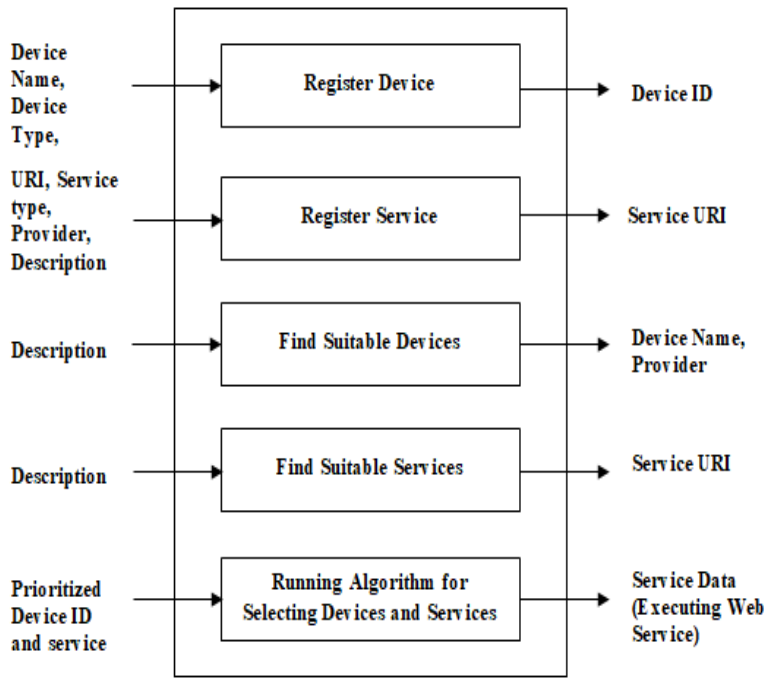

Figure 2: Internal architecture of the IoT service broker

A broker is the backbone architecture and is responsible for managing device and service registration, discovery of device and services and retrieving the resources for the services. Architecture ensures that resources of IoT devices and services available to the user. It includes an API that enables extractions of resource description of IoT devices and services which are registered and available in the cloud. The descriptions such as device details, service details and web service for the available services are stored in the database which is configured within the cloud.

Figure 2 represent the internal architecture of the IoT service broker.

\section{B. Module Description within the Broker}

Broker architecture allows several functionalities such as registration of devices and services, discovery of devices and services and selection of devices and services. These functionalities are developed and exposed using web services. Home page function is required to list different functionalities of broker architecture.

- Device Registration: The provider of the device enters the details of the device and registers to the framework. Once the device got registered, a web service is created for the device to generate the random value for the device. This random value indicates the sensor data.

- Service registration: Service providers can register their services to the framework and it uses cloud to store the entered data. A web service is created for service to analyse the sensor data.

- Device discovery: The users can easily identify the registered devices in the cloud by using device discovery module. Serach uses keyword matching to provide the reults to the user. From the device discovery user will get the details of the device which is stored in the cloud.

- Service discovery: Services which were already available in the cloud can be identified by using service discovery module. Search uses keyword matching to provide the reults to the user. From the service discovery user can identify the URI of the web service created for the service.

- Selecting device and service: The service requester enters the query to find particular service data, the query so keyed in by the user passes through several stages of algorithm. The algorithm contains several stages such as tokenization, stop word analysis, lemmatization and sentence filtering.

- In the first step, the algorithm collects descriptions of devices and services from cloud and English stop words from library.

- In next step tokenization, the entered query is considered as sentence and it splits sentence into separate words.

- Later it checks each word with stop words to filter the sentence. Here it removes all the stop words and stores the remaining words. This process is called as stemming.

- Then it considers synonyms for each word to collect all type of meanings and it brings contextual meaning to the words. This is called as lemmatization and it links words with similar meaning to one word. For lemmatization it considers words from WordNetLemmatizer. These processes take place for both user entered query as well as for descriptions which were already stored in cloud.

- After lemmatization in description and user entered query, the algorithm chooses the pair with highest path similarity among all the pairs. Out of that, the match with the maximum similarity is chosen. By considering maximum similarity, the algorithm analyses chosen device data and provides relevant information to user.

\section{Algorithm for Selecting Devices and Services using Semantic Search}

Input: Description, Date, Time from and Time to

Output: Generate data by binding suitable device and service to the user entered query

Steps:

1: Enter the description

2: Collects the descriptions of device and service

3: Collects stop words from English

4: Lemmatization

4a: Tokenize each description

4b: words in tokenized description is considered

If words are not in stop words

It checks for presence of symbols in words

Then add to filtered sentence 1

5: In filtered sentence1 it adds lemmatization words. For lemmatization, it considers the words from WordNetLemmatizer.

6: Repeat step 4 for user entered query

7: In filtered sentence 2 it adds lemmatization words of user entered query.

8: for each word from lemmatization word set1 stores synonym words from wordnet

9: for each word from lemmatization word set2 stores synonym words from wordnet 
10: calculates the similarity with each description and stores it in array.

11: Find the maximum similarity by arranging the words in the array in descending order.

12: By considering maximum similarity finds the device and service required for the query.

13: Run the web service to get the sensor data for the selected device and service.

14: The data corresponding to the requested device and service are retrieved from the cloud and returned to the service requester along with the service level.

15: If the description does not match, then the requested device is not registered.

\section{Implementation Method and Example}

At the beginning, a web service is created for all the services using PHP in Godaddy cloud server. A data base is created in cloud which receives the sensor data sent from the web services. The implementation is done by using broker based architecture by considering five different modules. The first two modules are used for device and service registration. The next two modules are used for finding the device and services in cloud. The last module is used for selecting devices and services. In selection of devices and services, the user entered query undergoes the process of semantic search.

Considering an example to represent the semantic search processing, if user enters the query as "How can I measure my blood pressure and glucose level"?

The algorithm collects descriptions of devices and services which are already stored in the cloud. It also collects English stop words from library by using library files. Pursuant thereto the entered query is split into separate words by way of tokenization. Further the stop words 'can', 'i', 'my', 'and' are filtered off and removed from the sentence above by the process of stemming and the residual words are retained. In the next stage, the architecture looks into the synonym to the residual words that are left behind after stemming and collects all types of meaning of the remaining words. The synonym words are considered from WordNetLemmatizer. Later on, the architecture chooses the pair with highest path similarity among the description of the devices, services and query entered. Finally, the match with the maximum similarity between the description of the device, service and the user query is chosen. By considering the proximity and similarity, the architecture will fetch and analyze the sensor data through web service and return the result to the user.

The equations are an exception to the prescribed specifications of this template. You will need to determine whether or not your equation should be typed using either the Times New Roman or the Symbol font (please no other font). To create multileveled equations, it may be necessary to treat the equation as a graphic and insert it into the text after your paper is styled.

\section{CONCLUSION AND FUTURE SCOPE}

The proposed IoT device and service discovery framework will allow the device and service providers to register the devices and services offered by them. In this framework user can check which and all devices and services already got registered into this framework. Also, the algorithm will fetch the suitable device and service for the query entered by the user by using sematic search. The framework uses semantic searching technique to search the appropriate data and will match and bind the suitable device with a compatible service will be correspondingly made and the appropriate data will be thus be made available to the user.

Therefore, proposed unified broker based architecture using web services which allows different providers to register their devices and services at one place. The user need not find for the devices and services separately. Instead he can find the registered devices and services at a single framework and binds the devices and services together to process the IoT device data stored on cloud.

In future, IoT applications would be deployed in several domains like home automation, smart cities, intelligent transport and e-health. Complex service request can also be made by the user which can be resolved using service composition i.e., by combining the available devices and service in all the available permutations and combinations and fetching different service data to get the final result.

\section{REFERENCES}

[1] V. Sharma and R. Tiwari, "A review paper on IOT and its smart applications", International Journal of Science, Engineering and Technology Research, pp.472-476, Feb 2016.

[2] S. R. Islam, D. Kwak, M. H. Kabir, M. Hossain and K. S. Kwak, "The Internet of Things for Health Care: A Comprehensive Survey", IEEE Access, 3, pp. 678-708, 2015.

[3] Dong, Hai, F. K. Hussain, and E. Chang. "A survey in semantic search technologies." 2008 2nd IEEE international conference on digital ecosystems and technologies, pp. 403-408, Feb 2008.

[4] M. Thiyagarajan and C. Raveendra, "Role of Web Service in Internet of Things", $3^{\text {rd }}$ International Conference on Applied and Theoritical Computing and Communication Technology, pp. 268-270, Dec 2017.

[5] E. Wang and R. Chow, "What can I do here? IoT service discovery in smart cities", IEEE International Conference on Pervasive Computing and Communication Workshops, pp. 1-6, Mar 2016.

[6] M. Aziez, S. Benharzallah and H. Bennoui, "Service Discovery for the Internet of Things: Comparison Study of the Approaches", $4^{\text {th }}$ International Conference on Control, Decision and Information Technologies, pp. 0599-0604, Apr 2017.

[7] S. K. Datta, C. Bonnet, A. Gyrard and R. P. F. Da Costa, "Applying Internet of things for Personalized Healthcare In Smart Homes", $24^{\text {th }}$ Wireless and Optical Communication Conference, pp. 164-169, Oct 2015.

[8] S. B. Fredj, M. Boussard, D. Kofman and L. Noirie, "Efficient semantic-based IoT Service Discovery Mechanism for Dynamic Environments", IEEE $25^{\text {th }}$ International Symposium on Personal, Indoor and Mobile Radio Communications, pp.2088-2092, Sep 2014.

[9] D. Georgakopoulos, P. R. Jayaraman, M. Zhang and R. Ranjan, "Discovery-Driven Service Oriented Iot Architecture", IEEE Conference on Collaboration and Internet Computing, pp. 142-149, Oct 2015.

[10] D. A. D’Mello, V. S. Ananthanarayana and S. Salian, "A Review of Dynamic Web Service Composition Techniques", International Conference on Computer Science and Information Technology, pp. 85-97, Jan 2011.

[11] S. Chirila, C. Lemnaru and M. Dinsoreanu, "Semantic-based IoT device discovery and recommendation mechanism", International Conference on Intelligent Computer Communication and Processing, pp. 111-116, Sep 2016. 
[12] F. Khodadadi, A. V. Dastjerdi and R. Buyya, "Simurgh: A Framework for Effective Discovery, Programming, and Integration of Services Exposed in loT", International Conference on Recent Advances in Internet of Things, pp. 1-6, Apr 2015.

[13] M. Aziez, S. Benharzallah and H Bennoui, "An Ontology based Context Model for the Discovery of IoT Services in the Internet of Things", International Conference on Mathematics and information Technology, pp. 209-213, Dec 2017.

[14] H. Moeini, I. L. Yen and F. Bastani, "Routing in IoT Network for Dynamic Service Discovery", $23^{\text {rd }}$ International Conference on Parallel and Distributed Systems, pp. 360-367, Dec 2017.

[15] J. Kim and J.W. Lee, "OpenIoT: An Open Service Framework for the Internet of Things", IEEE world forum on internet of things, pp. 89-93, Mar 2014.

[16] Pandiarajan, Sudhakar, and V. M. Yazhmozhi. "Semantic Search Engine Using Natural Language Processing." Advanced Computer and Communication Engineering Technology. Springer, Cham, pp. 561-571, 2015.

[17] Ma, Chao, M. Song, K. Xu, X. Zhang, "Web Service discovery research and implementation based on semantic search engine." 2010 IEEE 2nd Symposium on Web Society. IEEE, pp. 672-677, Aug 2010.

[18] El-gayar, M. M., N. Mekky, and A. Atwan. "Efficient proposed framework for semantic search engine using new semantic ranking algorithm." International Journal of Advanced Computer Science and Applications 6.8, Aug 2015. 